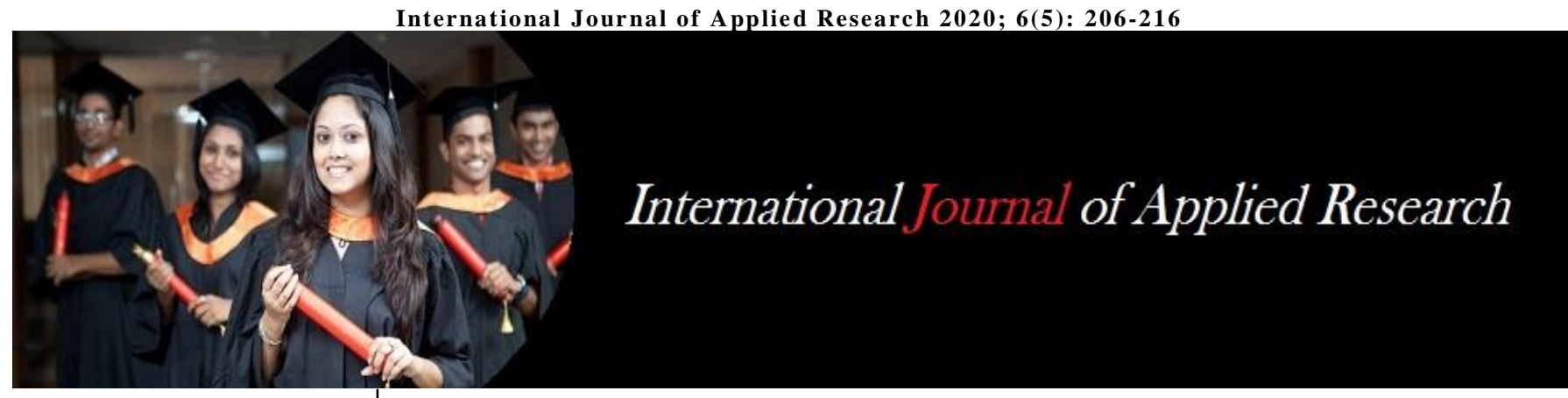

ISSN Print: 2394-7500 ISSN Online: 2394-5869 Impact Factor: 5.2

IJAR 2020; 6(5): 206-216 www.allresearchjournal.com

Received: 08-03-2020 Accepted: 10-04-2020

Maître Edmond Mbokolo Elima

(1) Assistant à la Faculté de Droit de l'Université de Mbandaka, République Démocratique du Congo (2) Avocat au Barreau près la Cour d'Appel de l'Equateur, République Démocratique du Congo

Correspondence Author:

Maître Edmond Mbokolo Elima (1) Assistant à la Faculté de Droit de l'Université de Mbandaka, République Démocratique du Congo (2) Avocat au Barreau près la Cour d'Appel de l'Equateur, République Démocratique du Congo

\section{L'exercice de la liberté de presse en Republique Democratique du Congo: Plaidoyer pour une dépénalisation des délits de presse}

\section{Maître Edmond Mbokolo Elima}

\section{DOI: https://doi.org/10.22271/allresearch.2020.v6.i5d.6703}

\section{Résumé}

La liberté de la presse, reste un droit d'informer en toute liberté et celui d'être informé tout aussi librement, découlant d'un parmi les droits fondamentaux de l'homme car prescrit tant par les instruments juridiques nationaux qu'internationaux. En République Démocratique du Congo, il est de constat irrévocable que, ce droit de l'homme est méconnu dans nos sociétés dites démocratiques car, dans la pratique de chaque jour, les libertés d'expression et d'opinion ne sont pas observées moins encore respectées, et les journalistes en sont victimes. D'où, la nécessité pour le législateur de dépénaliser les délits de presse afin de permettre aux acteurs médiatiques d'exercer leur profession en toute quiétude.

Mots-clefs: Droits de l'homme, journaliste, droit de la presse, droit d'informer, depenalisation, delit de presse, liberte de presse, journaliste en danger, plaidoyer

\section{Introduction}

La liberté de la presse est l'une des principales libertés publiques. C'est une condition nécessaire de l'exercice de la démocratie. Elle participe du droit d'expression et de critique dont disposent tous les citoyens vivant dans les pays démocratiques. Mais l'obtention de ce droit a demandé de longs combats. Aujourd'hui encore, cette liberté ne concerne qu'une minorité de pays. Cependant, même dans les pays démocratiques, la liberté de la presse doit composer avec les réalités économiques.

La constitution de la RDC, en tant que loi fondamentale, garantie la liberté d'expression et d'opinion ainsi que le droit à l'information. D'ailleurs, dans le préambule, elle affirme ce qui suit: "réaffirmant notre adhésion et notre attachement à la déclaration universelle des droits de l'homme, à la charte africaine des droits de l'homme et des peuples » ${ }^{[1]}$.

Par ailleurs, la liberté d'expression s'entend du droit d'opinion et de la liberté de recevoir ou de communiquer des informations ou des idées sans qu'il puisse y avoir ingérence des autorités publiques et sans considération de frontière.

Considérée comme quatrième pouvoir, la presse joue un rôle fondamental d'organe de surveillance des agissements des représentants du pouvoir public et de leurs collaborateurs. Les médias dans ce cadre agissent comme des acteurs isolés des mouvements sociaux mais, deviennent aussi un contre-pouvoir terrifiant à travers leur stratégie d'information et de dénonciation ${ }^{[2]}$. Dans ce rôle de sauvegarde des principes et valeurs démocratiques, les médias agissent également comme acteurs à part entière des mouvements sociaux.

C'est une vérité aujourd'hui que la puissance de la presse dans la formation de l'opinion publique est indéniable. Son activité est susceptible d'accroître la capacité de chaque citoyen à comprendre, à analyser et à discuter librement des questions politiques, économiques et. socioculturelles. En cela, la mission de la presse devrait bénéficier, à l'instar de l'éducation ou de la santé, d'une attention particulière auprès des gouvernants. Elle rend indéniablement un service d'intérêt public. Ces tentatives de définitions de l'intérêt public l'illustrent.

\footnotetext{
1 Préambule de la Constitution de la RDC du 18 février 2006 telle que révisée par la loi n $11 / 002$ du 20 janvier 2011 portant révision de certaines articles de la Constitution de la RDC du 18/09/2006.

2 E-N.EVEU, Sociologie des mouvements sociaux, La découverte, Paris, 3 ème édition, 97.
} 
La liberté d'expression est garantie par l'article 23 de la Constitution congolaise sus-rappelé, mais également par l'article 24 qui prévoit que l'Etat doit favoriser un environnement médiatique pluraliste. Afin de mettre en œuvre cette obligation, le Conseil Supérieur de l'Audiovisuel et de la Communication (CSAC) a été créé par une loi de janvier $2011^{[3]}$ et a été effectivement mis en place en août 2011. La loi de janvier 2011 autorise le CSAC, autorité compétente pour toute décision en matière de presse, à étudier toute violation de la loi sur la presse.

Cependant, il demeure évident que la RDC est un Etat qui ne favorise pas l'épanouissement de la liberté de la presse. En effet, bon nombre de lois perpétuent l'encombrement de la presse en matière de diffusion des faits quotidiens. Des journalistes sont souvent arrêtés et détenus, les maisons d'édition et autres organes de presse indépendants sont l'objet de perquisitions ou suspensions fréquentes, et les diffuseurs de médias voient leurs signaux de retransmission interrompus de façon arbitraire et sans préavis ${ }^{[4]}$.

Par conséquent, la RDC figure régulièrement sur la liste des pays où les médias sont intentionnellement asphyxiés et il n'existe malheureusement aucun doute que les lois congolaises ne sont pas à la hauteur du standard international concernant une régulation démocratique des médias ${ }^{[5]}$.

Depuis plusieurs années, le problème est le même. Les journalistes sont arrêtés arbitrairement par des fonctionnaires de certains organismes sécuritaires étatiques et traités en dehors du circuit judiciaire normal. Ils sont humiliés, agressés, torturés et placés dans des cachots sans lien avec le parquet. Ils sont généralement privés, non seulement de leur liberté physique, mais de celle d'organiser leurs moyens de défense. Leur libération ne dépend que de l'avis des responsables de ces organismes qui, dans le meilleur des cas, attendent les injonctions du pouvoir politique.

Le CSAC est l'instrument derrière lequel le ministre de tutelle peut se cacher pour sanctionner la presse qui dérange. Tirant les conséquences de cette situation catastrophique pour la régulation des médias, le Journaliste en Danger (JED) avait recommandé, dans son rapport annuel 2011, la dissolution pure et simple de cette structure et son remplacement par un nouvel organe de régulation plus crédible et compétent ${ }^{[6]}$.

Pour la RDC, malheureusement, et en dépit des garanties constitutionnelles affirmant la liberté de la presse, corolaire de la liberté d'expression et d'opinion, les années se suivent et ont tendance à se ressembler négativement. Des Journalistes menacés, emprisonnés, agressés, battus, parfois assassinés ; des médias fermés, attaqués à l'arme, parfois brûlés, ... tout cela fait partie du paysage politique congolais depuis bien longtemps et ne semble plus impressionner grand monde. Ce rapport en donne des illustrations et affiche des statistiques, des noms des victimes, des dates et

\footnotetext{
3 Loi $\mathrm{n}^{\circ}$ 11/001 du 11 janvier 2011 portant composition, attribution et fonctionnement du Conseil Supérieur de l'Audiovisuel et de la Communication et Ordonnance présidentielle n ${ }^{\circ} 11 / 054 \mathrm{du} 12$ août 2011.

4 Republique Democratique Du Congo, Manuel traitant du droit des médias en Afrique australe, 2:2, in [en ligne] http//:www.kas.de, consulté le 06 janvier 2016.

5 Ibid.

6 Journaliste En Danger, L'état de la liberté de la presse en Afrique centrale francophone: la RDC sous prétexte de la guerre, Rapport Annuel, 2012, s.d, 10, in [en ligne] «http://afrikarabia.com/wordpress/wp-content/uploads/2014/01/JEDRapport-2012.pdf », consulté le 06 janvier 2016.
}

des lieux où se commettent des pires exactions contre les journalistes et contre les médias ${ }^{[7]}$.

Ainsi donc, appelés à travailler dans un environnement qui leur est hostile à tous points de vue, politique, économique et sécuritaire, les journalistes congolais semblent se résoudre à faire du "service minimum" c'est-à-dire juste ce qu'il faut pour ne pas prendre des risques et continuer à survivre, la main tendue vers ceux qui les nourrissent.

D'où cette terrible impression que les médias congolais ont cessé d'être un pouvoir, le quatrième pouvoir, pour devenir de simples caisses de résonnance des idéologies et des opinions même les plus ubuesques; que les chevaliers de la plume ou du micro, ne sont plus devenus que des petits soldats qui obéissent aux ordres des donneurs d'ordre politique ou des pouvoirs d'argent. Tout ceci au plus grand dam de la liberté de la presse et de la démocratie.

Face à cette recrudescence du respect de la liberté de presse en RDC, il est nous reviens de savoir le pourquoi du nonrespect de la liberté de presse, corollaire du droit à l'expression, en tant qu'un droit fondamental en République Démocratique du Congo? Dans cette perspective, n'est-il pas possible au législateur congolais de dépénaliser les délits de presse pour la garantie de ce droit de l'homme en faveur des professionnels de médias?

La réponse à ces deux interrogations fait l'objet de notre étude qui s'articule, hormis l'introduction et la brève conclusion, autour de deux points principaux traitant respectivement de l'exercice de la liberté de presse en RDC (I) et de la dépénalisation des délits de presse (II).

\section{L'exercice de la liberté de presse en RDC}

L'exercice de la liberté de presse en République Démocratique du Congo nous renvoie à cerner d'abord la notion de la liberté de presse (I.1), de parler sur les organes chargés d'assurer le respect de ladite liberté (I.2), de faire la nomenclature des textes juridiques sur la liberté de presse (I.3), et enfin, faire un état des lieux de la liberté de presse en RDC.

\section{I.1. Liberté de la presse}

\section{A. Définition de la liberté de la presse}

La liberté de la presse ${ }^{[8]}$ est l'une des principales libertés publiques. C'est une condition nécessaire à l'exercice de la démocratie. Elle participe du droit d'expression et de critique dont disposent tous les citoyens vivant dans les pays démocratiques. Mais l'obtention de ce droit a demandé de longs combats. Aujourd'hui encore, cette liberté ne concerne qu'une minorité de pays. Cependant, même dans les pays démocratiques, la liberté de la presse doit composer avec les réalités économiques. Elle est l'un des principes fondamentaux des systèmes démocratiques qui repose sur la liberté d'opinion, la liberté mentale et d'expression [9]

Selon l'article 8 de la loi n $96-002$ du 22 juin 1996 fixant les modalités de l'exercice de la liberté de presse, celle-ci est entendue comme le droit d'être informé, d'avoir ses opinions, ses sentiments et de les communiquer sans aucune entrave, quel que soit le support utilisé, sous réserve du

\footnotetext{
Journaliste En Danger, La liberté de la presse pendant les élections. Des medias en campagne, Rapport spécial annuel de 2011, s.d, 7-8.

8 La liberté de presse, in

«http://www.larousse.fr/encyclopedie/divers/liebrtédelapresse», consulté le $27 / 01 / 2016$.

9 Wikipedia, "La liberté de presse », in http://fr.wikipedia.org, consulté le 27/01/2016.
} 
respect de la loi, de l'ordre public, des droits d'autrui et des bonnes mœurs ${ }^{[10]}$.

\section{B. Eléments constitutifs de la liberté de la presse}

Par des éléments constitutifs de la liberté de la presse, cela nous pousse à comprendre les différentes notions qui composent et accompagnent l'exercice de cette liberté. Le présent point analyse ces éléments, du point de vue des professionnels de la presse d'une part, et d'autre part, du point de vue du public.

\section{B.1 La liberté de la presse du point de vue des professionnels de la presse}

Par professionnel de la presse, il faut entendre toute personne œuvrant au sein des catégories de métier et se vouant d'une manière régulière à la collecte, au traitement, à la production, à la diffusion de l'information et des programmes, à travers un organe de presse et qui tire l'essentielle de ses revenus de cette profession. De cette définition, il ressort quelques éléments constitutifs ci-après :

\section{- La collecte des informations}

Elle marque le point de départ de l'exercice de la liberté l'expression par les professionnels des médias. Elle consiste à recueillir des faits sociaux de tout genre en vue de leur traitement ${ }^{[11]}$.

\section{- $\quad$ Le traitement des informations}

Lorsqu'un journaliste collecte les informations, celles-ci sont toujours dans une situation brute. Ainsi, en vue d'éviter la déformation des faits, il est tenu de leur donner un sens plus réaliste ; et ce, en utilisant parfois l'euphémisme pour une bonne production.

\section{- La production de l'information}

Elle permet aux professionnels des médias en général ; et aux agences de presse en particulier, d'assurer la réalisation matérielle des informations collectées en vue de leur diffusion. La production des informations peut être réalisée à titre onéreux aux fins de leur diffusion.

\section{- La diffusion des informations}

La diffusion des informations est le droit de propager des faits ainsi que des idées collectées. Il est le corollaire de la liberté de pensée ${ }^{[12]}$; en ce sens qu'elle consiste à dire ce que l'on pense ou ce que l'on sait, en privé ou dans les médias. L'élément clé qui apparait, parmi les éléments cités à titre indicatif, est l'information.

\section{- L'information}

Etymologiquement, l'information vient du mot latin "informare », façonner, former... L'information est l'action d'informer, de s'informer, de donner la connaissance d'un fait ou de la rechercher. Il s'agit ici à titre exemplatif, la presse d'information.

En informatique, l'information désigne, l'élément conceptuel qui permet le traitement, le stockage et le traitement de connaissance. En d'autres mots, « tout ce qui peut être traité, stocké ou conserver dans l'ordinateur ${ }^{[13]}$.

\footnotetext{
${ }^{10}$ Article 8 de la loi ${ }^{\circ} 96-002$ du 22 juin 1996 fixant les modalités de l'exercice de la liberté de presse.

${ }^{11}$ Lire à ce propos l'article 1 du code de déontologie et éthique des journalistes congolais.

12 Article 22 de la Constitution de la RDC du 18 février 2006 telle que modifiée par la loi $\mathrm{n}^{\circ} 11 / 002$ du 20 janvier 2011 portant révision de certaines articles de la Constitution de la République Démocratique du Congo du 18 février 2006.

${ }^{13}$ E. Mbokolo Elima, Cours d'initiation à l'informatique, Mission Phoenix, Mbandaka, 2013
}

Par ailleurs, en télécommunication, l'information est un élément de connaissance (voix, donnée, image) susceptible d'être conservé, traité ou transmis à l'aide d'un support et d'un mode de codification normalisé ${ }^{[14]}$.

En communication, l'information est entendue comme des faits, des données ou des messages de toutes sortes mis à la disposition du public par voie de la presse écrite ou de la communication audiovisuelle ${ }^{[15]}$.

- Le droit à l'information ${ }^{[16]}$

Le droit à l'information qui autrefois était une théorie soutenue par la doctrine, est actuellement consacré par la Constitution de la RDC du 18 février 2006 telle que révisée à ce jour. S'analysant comme l'un des éléments constitutifs de la liberté de la presse, du point de vue du journaliste ou professionnel de la presse, le droit à l'information implique la prérogative d'informer. L'Etat a l'obligation d'assurer et de rendre effectif ce droit.

Cependant, dans la collecte, le traitement, la production ainsi que la diffusion de l'information, le journaliste doit facilement accéder aux sources.

\section{- Le libre accès aux sources d'informations}

$\mathrm{Au}$ nom de la liberté de la presse, le journaliste est libre d'accéder à toutes les sources d'informations ${ }^{[17]}$. Par source d'informations, il faut entendre l'origine des faits, des données ou des messages auxquels le journaliste se réfère pour mettre les informations à la disposition du public. Le libre accès aux sources d'informations implique le devoir de vigilance. En effet, le devoir de vigilance de la presse correspond aux droits particuliers de cette dernière. En d'autres mots, elle doit fournir des informations qui soient les plus véridiques possibles. Le professionnel de la presse est ainsi tenue de vérifier, avant de les diffuser, toutes les informations eu égard à leur véracité, leur contenu et leur origine.

\section{B.2 Du point de vue du public}

La liberté de la presse, du point de vue du public, est constituée du droit à l'information. Mais ce dernier s'analyse comme le droit du public d'être informé ${ }^{[18]}$. Le public a donc le droit de recevoir une information de qualité ; c'est-àdire l'information qui respecte l'ordre public et les bonnes mœurs. Cela ressort clairement de l'article 9 alinéa 8 de la loi organique portant composition, attribution et fonctionnement du CSAC.

En effet, dans ses attributions, le CSAC est appelé, notamment, à veiller à la qualité des productions des médias du secteur de l'audiovisuel tant public que privé et en promouvoir l'excellence ${ }^{[19]}$.

Avec l'avancée de nouvelles technologies de l'information et de la communication, l'Internet, via les réseaux sociaux, s'est ajouté à la liste des médias. Vu les effets pervers de ce dernier Internet, le législateur a confié au CSAC la mission

\footnotetext{
14 Article 22 de la Constitution de la RDC.

${ }^{15}$ Article 6 de la loi n6-002 du 22 juin 1996 fixant les modalités de l'exercice de la liberté de presse.

${ }^{15}$ Article 3 de la loi organique $\mathrm{n}^{\circ} 11 / 001 \mathrm{du} 10$ janvier 2011 portant composition, attribution et fonctionnement du Conseil Supérieur de l'Audiovisuel et de la Communication.

${ }^{16}$ Article 24 de la Constitution de la RDC.

${ }^{17}$ Article 11 de la loi fixant les modalités d'exercice de la liberté de presse.

18 Article 24 de la Constitution de la RDC.

${ }^{19}$ Article 9 alinéa 8 de la loi organique portant composition, attribution et fonctionnement du CSAC.
} 
de prendre toutes les mesures nécessaires en vue de protéger les enfants des effets néfastes et pervers de l'Internet ${ }^{[20]}$.

\section{Typologie et nature d'atteintes à la liberté de presse [21]}

Sur le plan international, il n'existe pas, à proprement parler, de typologie uniforme des atteintes à la liberté de la presse. Le JED [22] a opté pour la typologie de Reporters sans frontières (RSF) dont il est membre et correspondant en RDC. Il y a neuf formes d'atteintes à la liberté de la presse que voici:

1. Journalistes tués ou assassinés: il s'agit des journalistes ou professionnels des médias qui sont tués dans l'exercice de leur profession ou à cause de celle-ci. Les journalistes assassinés sont ceux qui ont été victimes des meurtres ciblés et délibérés, les visant personnellement dans le but de les faire taire définitivement.

2. Journalistes portés disparus: est considéré comme «porté disparu » tout journaliste dont on n'a plus des nouvelles et qu'il y a l'évidence que cette disparition est liée à son travail ou à sa qualité de journaliste. Et aussi considéré porté disparu, un journaliste dont tout indique qu'il a été tué mais aucun corps ou aucune preuve ne peut l'étayer de façon absolue. Un journaliste passé dans la clandestinité pour sa sécurité ou refuse volontairement de donner de ses nouvelles ne peut être considéré comme «porté disparu ».

3. Journaliste en prison: il s'agit des journalistes se trouvant en prison au moment de la rédaction du rapport. Ces journalistes ont été jetés en prison dans l'exercice de leur profession ou à cause de celle-ci.

4. Journalistes incarcérés : il s'agit de tout journaliste ayant été privé de sa liberté pendant plus de 48 heures pour des motifs liés à sa profession. Le lieu de la détention importe peu.

5. Journalistes interpellés : il s'agit de tout journaliste ayant été privé de sa liberté, pour des raisons professionnelles, pendant moins de 48 heures.

6. Journalistes agressés ou torturés: il s'agit de tout journaliste agressé physiquement, malmené, violenté, blessé, battu ou ayant subi des souffrances morales ou physiques extrêmes et délibérées en raison de ses opinions ou ses activités professionnelles.

7. Journalistes menacés ou harcelés: il s'agit des journalistes victimes de toute menace directe ou indirecte, collective ou individuelle, du fait de l'Etat ou de ses représentants, des formations politiques, des groupes armés ou mouvements rebelles, de communautés religieuses, etc. visant à porter un préjudice ou à entraver l'exercice de la profession du journaliste.

8. Les pressions juridiques, administratives ou économiques: il existe des pressions administratives qui s'observent le plus souvent dans les médias par des suspensions, mutations, révocations, etc. sous des

\footnotetext{
${ }^{20}$ Article 9 alinéa 20 de la loi précitée.

${ }^{21}$ WIKIPEDIA, « La liberté de presse », Loc.cit.

${ }^{22} \mathrm{http}: / / \mathrm{www} . j \mathrm{j}-$

afrique.org/index.php?option=com_content $\&$ view=article $\&$ id=185:rappo rt-annuel-2015\&catid=97:rapports\&Itemid=1695, consulté le 05 mai 2017.
}

motifs plausibles mais qui cachent une volonté de réduire au silence un journaliste.

9. Il y a des médias qui sont victimes des pressions économiques comme le chantage pour l'accès à la publicité, les contrôles fiscaux, etc. Souvent, le lendemain de la publication d'un article ou la diffusion d'une émission qui dérange que le service de fisc ordonne le scelle d'un média.

10. Entrave à la libre circulation nationale ou internationale de l'information : il s'agit de l'interdiction de paraître ou d'émission; les suspensions provisoires et les saisis des journaux dans les kiosques ou sur la rue; la confiscation des notes, casettes, pellicules, disquettes ou tout autre support d'information; les interdictions dans les ports et aéroports, de quitter le pays avec les journaux locaux; le refus de visa ou d'accréditation pour la presse étrangère et les correspondants locaux des médias étrangers; le non accès de certains journalistes ou médias à certaines manifestations publiques et le plasticage des maisons de presse, des messageries ou imprimeries.

\section{I.2. Les organes charges d'assurer le respect de la liberté de presse}

Parler des organes chargés d'assurer le respect de la liberté de presse en RDC, nous renvoi à étudier tour à tour le Conseil Supérieur de l'Audiovisuel et de la Communication (2.1), l'Union Nationale de la Presse Congolaise (2.2), et enfin, le Journaliste en Danger (2.3).

\section{a) Le Conseil Supérieur de l'Audiovisuel et de la Communication}

\section{- Objet, nature et siège du CSAC}

Le Conseil Supérieur de l'Audiovisuel et de la Communication, CSAC ${ }^{[23]}$ en sigle, est une institution d'appui à la démocratie. Il est indépendant, autonome et doté de la personnalité juridique. Son siège du Conseil est établi [24] à Kinshasa, capitale de la République Démocratique du Congo. En cas de circonstances exceptionnelles empêchant le Conseil de se réunir à son siège habituel, l'Assemblée plénière peut décider du lieu qui abritera provisoirement ses travaux. Le siège du Conseil ainsi que ses bureaux de représentation en province sont inviolables, sauf dans les cas prévus par la loi et dans les formes qu'elle prescrit.

\section{- Fonctions principales du CSAC}

Quoique la RDC ne possède qu'une seule autorité contrôlant la diffusion et la distribution des signaux et que différentes lois établissent certaines autorités de régulation, il reste cependant clair que le véritable pouvoir de contrôle sur les médias réside auprès de l'autorité exécutive du gouvernement; plus précisément auprès du Ministre de l'Information et des medias. En effet, le travail du Conseil Supérieur de l'Audiovisuel et de la Communication au côté de l'Autorité de régulation, lequel contrôle les aspects techniques de la diffusion médiatique, demeure éclipser par les attributs ministériels exercés par le pouvoir exécutif malgré la conception constitutionnelle du Conseil.

Ce qui nous renvoi à étudier les fonctions principales en tant que Conseil Supérieur de l'Audiovisuel et de la

\footnotetext{
${ }^{23}$ Article 2 de la loi organisant le CSAC et Manuel traitant du droit des médias en Afrique australe, République Démocratique du Congo, 1:12.
} $\sim 209 \sim$ 
Communication d'une part, et d'autre, par ses fonctions en tant qu'autorité de régulation.

\section{Fonctions en tant que CSAC}

Les articles 8 et 10 de la Loi $n^{\circ} 11 / 001$ décrivent les fonctions principales ${ }^{[25]} \mathrm{du}$ CSAC comme suit:

- garantir la liberté de la presse, de l'information et des moyens de communication des masses ;

- faire respecter la déontologie en matière d'information ;

- veiller sur l'accès équitable des partis politiques, des associations et de toute autre personne aux moyens officiels d'information et de communication ;

- élaborer son Règlement intérieur ;

- assurer une médiation en cas de conflit dans le domaine des médias ;

- promouvoir l'excellence des productions médiatiques ;

- veiller à la diffusion de la culture de la paix, de la démocratie, des droits de l'homme et des libertés fondamentales ;

- valoriser la culture nationale à travers les médias ;

- veiller à la protection de l'enfant ;

- présenter un rapport périodique et annuel ainsi que des avis techniques au Parlement sur les projets ou propositions de lois relatifs aux médias.

\section{Fonctions en tant qu'autorité de régulation}

Selon l'article 8 de la loi cadre $n^{\circ} 013-2002$ du 16 octobre 2002 sur les télécommunications en République Démocratique du Congo, l'autorité de régulation est un service public dotée de la personnalité juridique. De ce fait, elle a pour attributions ${ }^{[26]} \mathrm{de}$ :

- veiller au respect des lois, des règlements et des conventions en matière des télécommunications ;

- instruire les dossiers de demande de concession, délivrer les autorisations, recevoir les déclarations, établir les cahiers des charges correspondant aux autorisations et veiller à ce que les obligations contractées par leurs titulaires soient respectées ;

- Procéder aux homologations requises par la présente loi;

- définir les principes d'interconnexion et de tarification des services publics de télécommunications ;

- gérer et contrôler le spectre des fréquences ;

- élaborer et gérer le plan national de numérotation ;

- analyser et étudier de façon prospective l'évolution, aux plans national et international, de l'environnement social, économique, technique et juridique des activités du secteur ;

- contribuer à définir et à adapter, conformément aux orientations de la politique gouvernementale, le cadre juridique général dans lequel s'exercent les activités relevant du secteur des télécommunications.

\section{Composition du CSAC}

Le Conseil est composé de quinze membres désignés de la manière suivante :

- un membre par le Président de la République ;

- deux membres par l'Assemblée nationale ;

- deux membres par le Sénat;

- un membre par le Gouvernement;

\footnotetext{
${ }^{25}$ Manuel traitant du droit des médias en Afrique australe, Op. cit., 30-31.

${ }^{26}$ Article 8 de la loi $n^{\circ} 013-2002$ du 16 octobre 2002 sur les télécommunications en République Démocratique du Congo.
}

- un membre par le Conseil Supérieur de la Magistrature ;

- trois membres par les associations des professionnels des médias, à raison d'un membre pour chaque secteur d'activité, à savoir : la radiodiffusion sonore, la télévision, la presse écrite ;

- un membre représentant du secteur de la publicité ;

- un membre par le Conseil national de l'ordre des avocats ;

- un membre par les associations des parents d'élèves et d'étudiants, légalement constituées;

- deux membres par les associations de défense des droits des professionnels des médias, légalement constituées.

Notons par ailleurs que, cette désignation tient compte de l'expertise dans le secteur des médias, de la représentation nationale ainsi que de celle de la femme.

\section{- Les organes et fonctionnement du CSAC}

Les organes du conseil sont:

1. L'assemblée plénière;

2. Le bureau;

3. Les commissions permanentes;

4. Les coordinations provinciales.

Pour l'Assemblée plénière ${ }^{[27]}$, elle est l'organe de décision du Conseil.

S'agissant du Bureau, c'est l'organe d'exécution et de gestion du Conseil. Il est composé de cinq membres, à savoir : un Président, un Vice- président, un Rapporteur, un Rapporteur adjoint et un Questeur. Sans préjudice d'autres attributions lui conférées par le Règlement intérieur, le Bureau élabore le projet du Budget du Conseil, conformément à la loi financière et le transmet au Gouvernement après son adoption

Par contre, les Commissions sont des organes permanents chargés de traiter des questions spécifiques ayant trait aux missions du Conseil.

Elles sont au nombre de trois, à savoir :

1. la Commission juridique chargée de l'enregistrement et de l'examen des plaintes, de la réglementation et des avis ;

2. la Commission technique chargée du contrôle de médias et de la conformité de la publicité;

3. la Commission socio-économique chargée des études, de la promotion et du développement de médias.

Salon l'article 49 de la loi sur la SCAC, la coordination provinciale est chargée de l'exécution des missions du Conseil en province. Elle est dirigée par un Coordonnateur, assisté d'un Coordonnateur adjoint.

Elle est dotée d'une extension du Centre de monitoring de médias congolais, du Secrétariat d'instruction et du Service de normalisation, et dispose d'un personnel technique et administratif.

\section{B. L'Union nationale de la presse congolaise Nature juridique}

L'Union nationale de la presse congolaise (UNPC) est une organisation indépendante de soutien aux journalistes en République Démocratique du Congo ${ }^{[28]}$. Sous un autre angle, l'UNPC est le seul syndicat des médias institué par la loi portant statut des journalistes.

Par ce fait du monopole légal, cette structure s'est dotée de beaucoup plus de pouvoirs dans la mesure où, aux dires de

\footnotetext{
${ }^{27}$ Article 34 de la loi sur le SCAC.

${ }^{28}$ Wikipedia, « Union nationale de la pesse congolais », in [en ligne]

« http://fr.wikipedia.org », consulté le 23 février 2016.
} 
certains experts, le contrôle exercé par elle est excessif, alors que sa mission première reste celle de garantir la liberté de la presse. Par exemple, dans une compréhension la plus simpliste de l'article 5, il est dit que seule l'UNPC est habilitée à émettre les cartes de presse alors que dans l'article 54 de la même loi, tout journaliste est libre de s'affilier à toute organisation professionnelle ou syndicale compatible et d'exercer ses activités aussi bien à l'intérieur de l'UNPC ou pas. Ce qui aboutit au fait que, d'après l'UNPC, cette dernière est la seule autorisée à octroyer la qualité de journaliste.

\section{Missions principales}

L'UNPC a pour missions ${ }^{[29]} \mathrm{de}$ :

- Coordonner les activités des groupements et associations membres ;

- Défendre la liberté de la presse, le droits et les intérêts généraux de la presse et de ses membres ;

- Organiser la profession, éditer les règles déontologiques et éthiques et en sanctionner les manquements ;

- Identifier les journalistes et assimilés opérant en République Démocratique du Congo ;

- Délivrer la carte de presse conformément à l'article 5 de l'ordonnance- loi $\mathrm{n}^{\circ} 81 / 012 \mathrm{du} 02$ avril 1981 portant statuts des journalistes œuvrant en RD du Congo ;

- Accréditer les journalistes étrangers en mission en RD du Congo ;

- Représenter la profession à l'intérieur comme à l'extérieur du pays ;

- Promouvoir la presse sur le plan moral, professionnel et matériel ;

- Renforcer la compréhension et la solidarité entre ses membres et établir, en son sein des liens entre diverses associations professionnelle de la presse en RDC ;

- Maintenir les contacts et promouvoir les échanges de vue entre la presse et les institutions publiques et privées ;

- Faciliter à ses membres l'accès aux sources d'information ;

- Organiser des activités de formation professionnelle et syndicale en faveur de ses membres.

\section{Le Journaliste En danger (JED) ${ }^{[30]}$ \\ - Brève présentation de JED}

Journaliste en danger (JED) est une organisation indépendante et non partisane de promotion et défense de la liberté de presse crée en 1998. JED est membre de IFEX (International Freedom of Expression Exchange), le plus grand réseau mondial des défenseurs de la liberté d'expression basé à Toronto (Canada). JED est aussi membre du Réseau International de Reporters sans frontières (RSF, Paris), une référence mondiale dans la défense de la liberté d'expression.

Depuis mai 2003, JED surveille également la liberté de la presse dans huit autres pays de l'Afrique centrale couverts par l'OMAC (Organisation des Médias d'Afrique Centrale dont le siège est à Bujumbura, capitale du Burundi. Les 8 autres pays sur lesquels s'étend le mandat de JED sont les suivants : Burundi, Cameroun, Congo/Brazzaville, Gabon,

\footnotetext{
${ }^{29}$ Missions de l'UNPC, in [en ligne] « http://www.unpc.info/article-517.html », consulté le 01 janvier 2016.

${ }^{30}$ Présentation de JED, in «http://www.jed-afrique.org/index.php? », consulté le 30 mars 2016.
}

Guinée Equatoriale, République Centrafricaine, Rwanda et Tchad.

JED est née d'un souci que ses fondateurs se sont fait au constat selon lequel, malgré la chute de la dictature du Maréchal Mobutu et la «libération» annoncée par l'avènement de l'AFDL (Alliance des forces démocratiques pour la libération) de Laurent-Désiré Kabila, il ne se passait plus aucun jour sans que, quelque part dans notre société, la liberté de la presse ne soit impunément violée, sans qu'une plume ne soit brisée.

Le constat était aussi que les journalistes étaient, souvent, victimes d'une justice sommaire. Tout détenteur d'une parcelle de pouvoir politique, économique ou militaire pouvant se rendre justice chaque fois que le discours de la presse ne l'agréait pas. Le journaliste était alors détenu en prison et son média était interdit de diffusion aussi longtemps que le voulait le bourreau.

Le trio fondateur de JED (Donat M'Baya, Tshivis Tshivuadi et Moro Mwamba), tous journalistes, s'était basé sur la valeur fondamentale de des droits de l'homme: $L a$ liberté. La liberté de la presse tire son fondement juridique de trois principaux instruments juridiques internationaux que la RDC a ratifié. Il s'agit de la Déclaration universelle des droits de l'Homme, du Pacte international relatif aux droits civil et politique et la Charte Africaine des droits de l'Homme et des peuples. La RDC a l'obligation de les respecter.

Pour bien surveiller au quotidien la liberté de la presse, JED a mis en place un réseau national d'alerte composé de plus de 250 correspondants. Ce sont ces correspondants qui sont l'œil et les oreilles de JED dans les provinces.

\section{- Des missions du JED}

JED se mobilise au quotidien pour:

- Surveiller l'exercice de la liberté de la presse dans les 9 pays de la sous-région Afrique Centrale par le monitoring et les enquêtes de terrain sur toutes les allégations d'atteintes à la liberté de la presse;

- Mener des actions de protestation (par des lettres ou alertes) et de lobbying auprès des responsables des atteintes à la liberté de la presse afin de faire cesser ces violations dans chacun des pays et/ou obtenir réparation;

- Effectuer des visites régulières sur les lieux de détention ou d'emprisonnement des journalistes;

- Attribuer (directement ou indirectement) une petite bourse d'assistance aux familles des journalistes tués ou emprisonnés en raison de l'exercice de leur profession ;

- Apporter une assistance judiciaire aux journalistes ou médias poursuivis devant les cours et tribunaux pour leur travail, par la mise à leur disposition des avocats.

- Observer les procès intentés contre les médias ou les journalistes ;

- Participer à des études sur l'amélioration du cadre juridique, politique et économique de l'exercice de la liberté de la presse ;

- Publier des rapports annuels sur la liberté de la presse ou sur des thèmes spécifiques en rapport avec l'actualité des médias, seul ou avec des partenaires ;

- Organiser des ateliers, conférences, séminaires sur des questions touchant à l'éthique et à la déontologie, au traitement professionnel de l'information, et à la sécurité des journalistes ;

- Publier un Magazine dénommé «Plume et Liberté » 


\section{- Les cinq programmes de JED}

1. Monitoring de la liberté de la presse : c'est la principale action de JED qui consiste à surveiller la liberté de la presse grâce à ses correspondants se trouvant dans les 11 provinces de la RDC et les pays de la sous de la sous-région.

2. La professionnalisation des médias : par ce programme, JED sensibilise les professionnels des médias par des ateliers, séminaires notamment sur l'éthique et la déontologie du journaliste, etc.

3. Défense légale des journalistes et des médias : il s'agit de l'assistance judiciaire des journalistes et des médias déférés devant les cours et tribunaux. Dans beaucoup de provinces, JED a signé des contrats avec des cabinets d'avocats pour la défense des journalistes poursuivis par la justice pour des raisons liées à leur profession.

4. Lobbying et plaidoyer : JED fait des lobbyings et des plaidoyers pour la réforme des lois qui régissent la presse en RDC. Tel est le cas de la loi $n^{\circ} 96 / 002$ du 22 juin 1996 fixant les modalités de l'exercice de la liberté de la presse en RDC qui date de la période de la dictature. JED fait également de plaidoyer pour la dépénalisation des délits de presse, la loi sur l'accès à l'information, etc.

5. Fonds d'urgence d'aide aux journalistes en danger : ce fonds est destiné à sécuriser les journalistes qui ont des problèmes. Ex : évacuation d'un lieu à un autre des journalistes menacés. Ce fonds n'est pas disponible. En cas de besoin, JED contacte en urgence ses bailleurs de fonds pour venir en aide au journaliste qui a des problèmes.

\section{I.3. Nomenclature des textes juridiques sur la liberté de presse en RDC ${ }^{[31]}$}

Les textes juridiques existant dans le domaine de la radiodiffusion peuvent être classés en trois grands groupes à savoir : ceux ayant une portée internationale, ceux ayant une portée strictement nationale et ceux ayant une portée quasiprivée du fait de la nature de leur émanation ${ }^{[32]}$.

\section{A. Textes ayant une portée internationale ${ }^{[33]}$}

Il s'agit de quatre textes essentiels qui, bien que ne s'appliquant pas exclusivement à la radiodiffusion, se réfèrent néanmoins à la liberté de la presse et donc à la radiodiffusion. Ces textes posent des principes universellement admis en matière de liberté d'expression, de liberté de la presse et du droit du public à l'information.

\section{La Déclaration Universelle des Droits de l'homme}

Adoptée par l'Assemblée Générale des Nations unies dans sa résolution 217 A (III) du 10 décembre 1948, la DUDH, dont la RDC est partie prenante pour l'avoir ratifiée, dispose en son article 19 que : « tout individu a droit à la liberté d'opinion et d'expression, ce qui implique le droit de ne pas être inquiété pour ses opinions et celui de chercher, de recevoir et de répandre, sans considération de frontières, les informations et les idées par quelque moyen d'expression que ce soit ».

\footnotetext{
${ }^{31}$ C-M. Mushizi, Le cadre juridique et institutionnel des médias congolais, Kinshasa, Décembre 2007, 16-17.

${ }^{31}$ Ibid, 17-19.

32 Ibid.
}

Cet article 19 garantit aussi la liberté de collecter, de diffuser des informations, ainsi que le droit pour d'autres. Il s'agit bien du public ou (du droit du public à l'information) de recevoir ces informations en toute liberté.

\section{Le Pacte international relatif aux Droits civils et politiques}

Adopté par la résolution $\mathrm{n}^{\circ} 2200 \mathrm{~A}(\mathrm{XXI})$ de l'Assemblée générale des Nations unies en sa session du 16 décembre 1966, le PIDCP est entré en vigueur le 23 mars 1976. La RDC l'a ratifié. Une de ses dispositions stipule que : «nul ne peut être inquiété pour ses opinions. Toute personne a droit à la liberté d'expression ; ce droit comprend la liberté de rechercher, de recevoir et de répandre des informations et des idées de toute espèce, sans considération de frontières, sous une forme orale, écrite, imprimée ou artistique, ou par tout autre moyen de son choix ».

\section{La Charte africaine des Droits de l'homme et des peuples}

Quinze ans après l'adoption du Pacte international relatif aux Droits civils et politiques, plus précisément le 27 juin 1981, les chefs d'États africains réunis au sein de l'OUA (Organisation de l'unité africaine, devenue l'Union africaine), invoquant les particularismes culturels de l'Afrique, adoptent la Charte africaine des Droits de l'homme et des peuples (CADHP).

L'article 9 de cette charte dit en deux alinéas : " toute personne a droit à l'information. Toute personne a le droit d'exprimer et de diffuser ses opinions dans le cadre des lois et règlements ».

\section{La Charte de munich}

En 1971, des journalistes originaires de six pays d'Europe de l'Ouest, réunis dans la ville de Munich en Allemagne, ont rédigé une déclaration communément appelée La Charte de Munich. Cette Charte qui comporte des devoirs et des droits des journalistes a, depuis, été adoptée par la Fédération Internationale des Journalistes (FIJ). Ce document constitue la matrice de la plupart des codes d'éthique et de déontologie des journalistes à travers le monde y compris ceux de la RDC.

\section{B. Textes ayant une portée nationale ${ }^{[34]}$ \\ - La Constitution de la republique democratique du Congo \\ La Constitution de la République Démocratique du Congo} telle que modifiée par la loi $\mathrm{n}^{\circ} 11 / 002$ du 20 janvier 2011 portant révision de certains articles de la Constitution de la République Démocratique du Congo du 18 février 2006 fonde et garantie, en sa qualité de la loi mère et fondamentale, les notions de la liberté d'expression, du droit à l'information, de la liberté de presse et de la liberté d'information et d'émission.

En son article 23, elle prévoit que : «toute personne a droit à la liberté d'expression. Ce droit implique la liberté d'exprimer ses opinions ou ses convictions, notamment par la parole, l'écrit et l'image, sous réserve du respect de la loi, de l'ordre public et des bonnes mœurs.

L'article 24 renchérit que : «toute personne a droit à l'information. La liberté de la presse, la liberté d'information et d'émission par la radio et la télévision, la 
presse écrite ou tout autre moyen de communication sont garanties sous réserve du respect de l'ordre public, des bonnes mœurs et des droits d'autrui. La loi fixe les modalités d'exercice de ces libertés. Les médias audiovisuels et écrits d'Etat sont des services publics dont l'accès est garanti de manière équitable à tous les courants politiques et sociaux. Le statut des médias d'Etat est établi par la loi qui garantit l'objectivité, l'impartialité et le pluralisme d'opinions dans le traitement et la diffusion de l'information ».

L'article 212 prévoit que «il est institué un Conseil supérieur de l'audiovisuel et de la communication dotée de la personnalité juridique. Il a pour mission de garantir et d'assurer la liberté et la protection de la presse, ainsi que de tous les moyens de communication de masse dans le respect de la loi. Il veille au respect de la déontologie en matière d'information et à l'accès équitable des partis politiques, des associations et des citoyens aux moyens officiels d'information et de communication ».

\section{- Loi n`96-002 du 22 juin 1996 fixant les modalités de l'exercice de la liberté de presse}

La loi $n^{\circ}$ 96-002 du 22 juin 1996 fixant les modalités de l'exercice de la liberté de presse, surabonde en disposant en son article 8 que : «toute personne a droit à la liberté d'opinion et d'expression. Par liberté d'opinion et d'expression, il faut entendre le droit d'informer, d'être informé, d'avoir ses opinions, ses sentiments et de les communiquer sans aucune entrave quel que soit le support utilisé sous réserve du respect de la loi, de l'ordre public et des droits d'autrui et des bonnes mœurs ».

\section{- Loi organique $\mathrm{n}^{\circ} 11 / 001$ du 10 janvier 2011 portant composition, attribution et fonctionnement du CSAC}

cette loi organique attribue expressément au CSAC, parmi ses missions prévues à l'article 8 , la compétence de l'autorité de régulation des médias, partant garant du respect scrupuleux de la liberté de la presse, de l'information et de tout autre moyen de communication des masses ainsi que d'assurer la protection de la presse. Il faut préciser encore que, cette loi organique, reconnait encore au CSAC, spécialement à l'article 9 point 6 , l'attribution de veiller au respect de la loi fixant les modalités de l'exercice de la liberté de presse en République Démocratique du Congo.

\section{Textes ayant une portée privée ${ }^{[35]}$ \\ - Codes d'éthique et de déontologie des journalistes congolais}

Un Code de déontologie et éthique du journaliste congolais a été mis en place afin que le droit à l'information, à la libre expression et à la critique soit l'une de libertés fondamentales de tout être humain et que de ce droit du public à contrarier les opinions, procèdent de l'ensemble des devoirs et des droits des journalistes.

De ce fait, l'article premier impose à tout journaliste travaillant sur le territoire congolais d'œuvrer en tout temps en faveur de la liberté dans la collecte, le traitement et la diffusion des informations, opinions, commentaires et critiques; cette liberté étant indissociable du droit public à être informé et à recevoir et émettre librement des opinions.

\footnotetext{
${ }^{35}$ C-M. Mushizi, Op. cit., 20-21.
}

\section{- Les rapports de JED sur les violations de la liberté} de presse en RDC

Il n'est point besoin de rappeler que le JED se mobilise au quotidien pour surveiller l'exercice de la liberté de la presse en RDC par le monitoring des atteintes au droit d'informer et d'être informé en toute liberté par les médias.

En effet, dans son rapport de 2012, le JED relève que : «le contexte politique et médiatique de la République démocratique du Congo a été marqué, durant l'année 2012, par deux événements majeurs à savoir : la crise politique qui a suivi les élections présidentielles et législatives du 28 novembre 2011 dont les résultats ont été fortement contestés par une large partie de l'opinion nationale et internationale, et l'éclatement, au mois d'avril 2012, d'une nouvelle guerre à l'Est du pays opposant les Forces loyalistes à un groupe de soldats mutins qui seraient soutenus, selon un rapport des experts des Nations unies, par le Rwanda voisin. Comme il fallait s'y attendre, ces deux événements ont créé un climat général de tension et d'insécurité propice à des graves violations des droits de l'homme et des libertés fondamentales, dont la liberté de presse et d'expression » [36]

Cette année, particulièrement dans l'Est de la RDC, les violations de la liberté des journalistes (interpellations, détentions arbitraires), les menaces sur leur sécurité, et les actes de censure envers les médias se sont multipliés depuis le mois d'avril.

Dans la même optique, le rapport annuel 2015 du JED renseigne que, «l'année 2015 qui s'achève aura été à n'en point douter, une année thermomètre, qui indique que le processus démocratique a enregistré une poussée de fièvre, et que la liberté d'expression est quelque peu " grippée » ainsi que tous les autres domaines de la vie sociale en R.D Congo. Dès le début de l'année précisément le 19 janvier des violences ont éclaté à Kinshasa, la capitale, où une manifestation à l'appel de l'opposition contre la réforme de la loi électorale a dégénéré, l'opposition redoutant que la réforme de la loi électorale soit une manœuvre de la Majorité au pouvoir visant à repousser de plusieurs années la tenue des élections prévues pour $2016 »{ }^{[37]}$.

Il précise sans ambages que, il ressort que sans surprise, la RDC occupe la $150^{\text {ème }}$ place sur 180 pays au classement mondial de la liberté de la presse publié par reporters sans frontières en février 2015. Et pour cause, année après année, le climat général du travail des professionnels des médias ne s'améliore guère ${ }^{[38]}$. La multiplication des actes de violence contre la presse, la censure des médias, et les actes d'intimidation contre les journalistes, démontrent que la RDC figure toujours parmi les pays du monde où il ne fait pas toujours bon d'être journaliste, aussi bien pour la presse nationale que pour les médias internationaux constamment confrontés à la stigmatisation.

Sur le plan des statistiques des atteintes à la liberté de l'information enregistrées cette année, depuis la publication du dernier Rapport de JED en novembre 2014, on compte : 2 journalistes tués, 22 journalistes privés de leur liberté pendant des périodes plus ou moins longues et 25

\footnotetext{
${ }^{36}$ Journalise En Danger, Etat de la liberté de presse en Afrique

Francophone. RD Congo: sous prétextes de la guerre..., Rapport Annuel 2012, 10 décembre 2012, 10.

37 Journaliste En Danger, Liberté d'expression et élections en RD CONGO,

2016: L'information sous haute surveillance, Rapport annuel 2015,

Kinshasa, Novembre 2015, 4-5. ${ }^{38}$ Ibid.
} 
journalistes agressés ou menacés dans l'exercice de leur profession ${ }^{[39]}$.

Nul ne doute que ces mesures gravissimes de répression contre les médias ont marqué un tournant décisif dans le processus démocratique en cours, et ont été, à ce point, révélatrices d'un état d'esprit fondamentalement hostile à la liberté de presse et d'expression dans le chef des gouvernants congolais.

\section{I.4. Etat des lieux de la liberté de presse en RDC}

La liberté de la presse, entendue comme le droit d'informer en toute liberté et celui d'être informé tout aussi librement, découle d'un droit de l'homme consacré par les instruments juridiques tant internationaux que nationaux sus-visés.

Le constat est amer dans la mesure où, la liberté d'expression et d'opinion, n'est pas respecté en RDC par les acteurs politiques, d'où à tout moment les journalistes sont arrêtés, portés disparus, les medias fermés, etc.......Alors qu'il existe une institution de régulation et ayant parmi ses missions la garantie et le respect scrupuleux de la liberté de pressé, il s'agit du SCAC.

A la lecture des différents rapports du JED, il est révélé qu'en 2015 par exemple, l'on trouve des cas de pressions et de censures exercées sur les médias et les journalistes par les pouvoirs publics représentent $38 \%$ d'attaques contre les médias, soit le record des violations. En outre, au moins 22 journalistes, soit $30 \%$ des professionnels des médias ont été privés de leur liberté pendant des périodes plus ou moins longues. Enfin, 25 cas des journalistes agressés ou menacés dans l'exercice de leur profession, ou des médias harcelés.

$\mathrm{Au}$ regard de ces chiffres, on peut noter que le métier d'informer demeure un métier à risque en RDC, et que les autorités congolaises n'ont pris aucune mesure, ni politique, ni administrative pour renforcer la sécurité des journalistes dans leur travail.

Il est à noter que, les atteintes à la liberté de la presse restent toujours très élevées en RDC. Malgré, selon JEJ, une légère diminution quantitative d'attaques dirigées contre les médias et les journalistes observée en 2015, la RDC présente encore des nombreux défis à relever pour la liberté de la presse et la sécurité des journalistes.

Par ailleurs, les différents gouvernements de terreur qui se sont succédés n'ont jamais pris aucune mesure positive allant dans le sens de l'amélioration de l'environnement de travail des journalistes, ou d'une meilleure sécurisation des professionnels des médias. L'impunité dont jouissent les personnes identifiables, responsables des violations, parfois flagrantes à la liberté de l'information constitue un obstacle majeur au travail des journalistes.

Car, dans toutes les années ciblées par notre étude, le problème est resté le même. Les journalistes sont arrêtés arbitrairement par des fonctionnaires de certains organismes sécuritaires étatiques et traités en dehors du circuit judiciaire normal. Ils sont humiliés, agressés, torturés et placés dans des cachots sans lien avec le parquet. Ils sont généralement privés, non seulement de leur liberté physique, mais de celle d'organiser leurs moyens de défense. Leur libération ne dépend que de l'avis des responsables de ces organismes qui, dans le meilleur des cas, attendent les injonctions du pouvoir politique.

D'où l'exercice de la liberté de presse en RDC, n'est pas garanti dans la pratique quotidienne, malgré la présence

\footnotetext{
${ }^{39}$ Journaliste En Danger, Rapport annuel 2015, Op. cit.
}

d'une ribambelle de textes juridiques y afférents et des instances de de régulations.

\section{Plaidoyer pour une dépénalisation des délits de presse en $\mathrm{RDC}$}

Il est question ici, de cerner la notion «délit de presse » (II.1), avant de se focaliser sur sa dépénalisation en droit congolais (II.2).

\section{II.1. Délit de presse, Quid?}

En RDC, lorsqu'on parle de « délit de presse », on doit vite recourir à l'article 74 de la loi n 96-002 du 22 juin 1996 fixant les modalités de l'exercice de la liberté de la presse, pour en tirer la définition. L'article cité de cette loi définit le délit de presse comme «toute infraction commise par voie de presse écrite ou audiovisuelle » ${ }^{[40]}$.

De cette définition, il ressort que chaque fois qu'une infraction aura été commise par voie de presse, c'est-à-dire par l'entremise d'un support médiatique écrit, électronique ou audio-visuel, il y aurait un délit de presse ${ }^{[41]}$.

Pierre AKELE décèle dans le chef du législateur congolais une vision très large du délit de presse du fait que celui-ci incrimine en quelque sorte « l'abus de la liberté de la presse, c'est-à-dire l'usage des médias à des fins de commettre quelque infraction que ce soit $\gg{ }^{[42]}$.

Ainsi, cette définition prévue dans l'article 74 de la loi du 22 juin 1996 est jugée vague à souhait par Léopold MBUYI, au point qu'on doive considérer le délit de presse plus comme un mode particulier de commission d'une infraction que comme une infraction propre. Les délits de presse dans cette loi, fait-il savoir, tiennent plus des circonstances aggravantes et des modes de participation criminelle que des infractions autonomes. Léopold MBUYI fait savoir que l'organisation de la responsabilité civile, la prescription et surtout la compétence y sont lacunaires. Cet oubli du législateur de l'époque n'était pas un hasard ${ }^{[43]}$.

Il faut noter que la notion de délit de presse n'est pas l'apanage du seul droit congolais. Jean-Marie CHARON et Claude FURET ${ }^{[44]}$ considèrent le délit de presse comme un délit d'opinion par le moyen de la presse. Pour eux, chaque fois que la presse sert de moyen d'expression à une infraction, cette infraction devient un délit de presse pour autant que la matière porte sur le délit d'opinion.

Pour sa part, Fernand TERROU ${ }^{[45]}$ note que la définition des délits de presse est inévitablement formulée en termes très généraux qui peuvent prêter à des interprétations, à des applications diverses et mouvantes. Dans le domaine de l'information, remarque-t-il, c'est du libéralisme du juge que dépend pour une large part le libéralisme du régime.

\footnotetext{
${ }^{40}$ Article 74 de la loi $n^{\circ}$ 96/002 du 22 juin 1996 sur la liberté de presse.

${ }^{41}$ Usard et Internews, Revue de la législation sur les Médias en République Démocratique du Congo, 'Programme de Développement du Secteur Médiatique (PDSM), Kinshasa, Juin 2012, 9-10.

${ }^{42}$ A. Akele Adau, « Dépénaliser les délits de presse en RDC : pourquoi et comment? », in Plaidoyer pour la dépénalisation des délits de presse en République Démocratique du Congo, Kinshasa, Mai 2004, cité par I. OLENGA LUMBAHEE, Op. cit.

${ }^{43}$ L. Mbuyi, «La problématique de la diffamation et des imputations dommageables par voie de presse », in Code de déontologie des journalistes en RDC, OMC, Kinshasa, février 2005, cité par Olenga Lumbahee, Op. cit.

${ }^{44} \mathrm{~J}-\mathrm{M}$. Charon et C. Furet, Un secret si bien violé : la loi, le juge et le journaliste, Paris, Seuil, 2000, cité par OLENGA LUMBAHEE, Op. cit.

${ }^{45}$ F. Terrou, Sociologie juridique e droit de l'information, Paris, Librairie Larousse, 1973, cité par I. Olenga Lumbahee, Op. cit.
} 
En définitive, tout fait, comme le font savoir Charles MUGAGGA MUSHIZI et Donat M'BAYA TSHIMANGA

${ }^{[46]}$ dès lors qu'il peut être interprété comme enfreignant une des dispositions pénales en vigueur en RDC ou dans l'ordre judiciaire international, constitue un délit de presse pour autant qu'il soit perpétré par voie de presse. Il engendre ainsi, automatiquement, une responsabilité pénale dans le chef de son auteur, même si les poursuites ne s'en suivent pas automatiquement ni immédiatement.

On pourrait donc déduire que, chaque fois que la presse sert de moyen d'expression à une infraction, cette infraction devient un délit de presse. Le délit de presse peut donc être le fait d'un professionnel des médias ou de n'importe quel individu qui se servirait d'un support écrit ou audiovisuel propre aux médias pour commettre une infraction. Ce qui limite la liberté des professionnels de medias à accomplir leur mission avec toute liberté, d'où la ratio legis de notre plaidoyer pour sa dépénalisation.

\section{II.2. La dépénalisation des délits de presse en RDC}

La dépénalisation désigne l'abolition de sanctions pénales pour certains actes, même si des amendes preuves toujours s'appliquer Cette évolution est différente de la légalisation. En dépénalisant, le rédacteur décide que soit l'infraction en cause reposera à l'avenir sur des conditions plus restrictives, soit exposera son auteur à une peine moins forte.

Dans une société démocratique, l'exercice d'une liberté, futelle le pilier de la défense des droits fondamentaux, ne peut se justifier par la commission d'infractions, à peine de contester la légitimité des règles d'ordre public, et par là même, du système tout entier ${ }^{[47]}$. Dans cette optique, remarque KOOVY YETE, il n'appartient pas à la presse ni de salir injustement l'honneur d'un homme, ni de publier par exemple des informations de nature à mettre en péril la défense nationale ${ }^{[48]}$. Tout en reconnaissant que la presse comme quatrième pouvoir est un atout nécessaire dans l'édification d'un Etat démocratique, MONTESQUIEU [49] prévient que ce pouvoir à l'instar de tout pouvoir devient dangereux et peut même ruiner le processus démocratique par l'inconscience de certains de ses membres.

Mais hélas, en démocratie ${ }^{[50]}$, nul ne peut être poursuivi pénalement pour ses opinions. Ceci est à la base de la norme anglo-américaine basée essentiellement sur la réparation des préjudices causés à autrui seulement par des dommagesintérêts, c'est-à-dire au civil pour les cas de diffamation.

Cela étant, presque partout dans le monde, les défenseurs de la liberté d'expression s'orientent vers un abandon pur et simple de l'aspect pénal lié à la diffamation au profit du recours aux dommages-intérêts. Dans certaines juridictions, comme celles aux États-Unis, les personnalités publiques se voient d'ailleurs accordées moins de protection que les personnes privées, et on exige en particulier de leur part de prouver une certaine gravité des faits reprochés au journaliste, telle que l'intention de nuire et la connaissance

${ }^{46} \mathrm{C}$. Mugagga et D. M'baya, Comprendre les textes juridiques et déontologiques régissant la presse en RDC, février 2006, Institut Panos Paris, p.69, cité par I. Olenga Lumbahee, Op. cit.

${ }^{47}$ P. Fontbressen, cité par Olenga Lumbahee, Dépénalisation des délits de presse en République Démocratique du Congo: analyse de l'action de journaliste en danger (JED). Approche sociologique du droit de l'information, Mémoire de Licence en Journalisme et Politique Intérieure, IFASIC-KIN, 2010.

${ }^{48}$ K.Yete, cité par Olenga Lumbahee, op.cit.

${ }^{49}$ Montesquieu, De l'esprit des lois, Livre III, Chap. V, 94, cité par Olenga Lumbahee, op.cit.

${ }^{50}$ Usaid ET Internews, Op. cit., 9-10. effective du caractère erroné des informations en cause, avant de gagner des dommages-intérêts.

Ce régime garantit une protection de qualité aux journalistes et renforce une discussion franche et ouverte des questions politiques dans la sphère publique ${ }^{[51]}$.

Raison pour laquelle, le législateur congolais devra dépénaliser les délits de presse en faveur des professionnels des médias et de toute autre personne se vouant à faire usage de son droit fondamental de la liberté d'expression.

Bref, il est impérieux au législateur de la RDC, d'édicter une nouvelle loi sur la dépénalisation de toutes les infractions commises par le biais des médias et de tout moyen de communication soit-il.

\section{Conclusion}

La liberté de la presse, reste un droit d'informer en toute liberté et celui d'être informé tout aussi librement, découlant d'un parmi les droits fondamentaux de l'homme car prescrit tant par les instruments juridiques nationaux qu'internationaux.

A l'heure actuelle, il est de constat irrévocable que, ce droit de l'homme est méconnu dans nos sociétés dites démocratiques car, dans la pratique de chaque jour, les libertés d'expression et d'opinion ne sont pas observées moins encore respectées en RDC.

Ce non-respect semble être imputé aux acteurs politiques ou pouvoirs publics, du fait qu'à tout moment les journalistes sont arrêtés, portés disparus, les medias fermés,... quand bien même qu'actuellement, on a connu la naissance d'un organisme public dit SCAC, ayant pour mission non seulement de réguler les médias mais aussi et surtout garantir le respect sans équivoque de l'exercice de la liberté de presse. Chose qui est reste monstre en RDC. D'où, les investigations et rapports des défenseurs de droits des journalistes, spécialement le Journaliste en Danger dévoilent qu'à titre illustratif, en 2015, il y a eu la présence irréfutable des cas de pressions et de censures exercées sur les médias et les journalistes par les pouvoirs publics représentent $38 \%$ d'attaques contre les médias, soit le record des violations.

De ce fait, le Gouvernement et le Parlement doivent respecter la liberté de la presse, corollaire de la liberté d'expression, telle que garantie par la loi sur la presse et la Constitution de la République Démocratique du Congo et multiples textes à caractère international. Ils doivent veiller à l'accès équitable et égalitaire de tous les acteurs aux médias publics.

Aussi, de militer pour la réouverture de tous les organes de presse fermés à travers toute l'étendue du pays, la libération de tous les journalistes qui sont en prison, l'accélération de l'adoption de la loi sur l'accès à l'information actuellement sous examen au Parlement ainsi que d'adopter un moratoire sur les arrestations et emprisonnements des journalistes, en attendant l'adoption d'une loi sur la dépénalisation des délits de presse.

\section{Notices bibliographiques \\ I. Textes légaux \\ 1.1. Internationaux}

1. Déclaration Universelle des Droits de l'Homme adoptée et proclamée par 1'Assemblée Générale des Nations unies dans la résolution 217 ACC III du 10 décembre 1948, B.O, 1949.

\footnotetext{
${ }^{51}$ Usaid et Internews, Op. cit., 9-10.
} 
2. Le Pacte international relatif aux Droits civils et politiques, Adopté par la résolution n ${ }^{\circ} 2200 \mathrm{~A}(\mathrm{XXI}) \mathrm{de}$ l'Assemblée Générale des Nations unies en sa session du 16 décembre 1966, entré en vigueur le 23 mars 1976.

3. La Charte africaine des Droits de l'homme et des peuples, Adopté le 27 juin 1981par les chefs d'États africains.

4. La Charte de Munich, adoptée par la Fédération internationale des journalistes (FIJ) en Munich (Allemagne), 1971.

\subsection{Nationaux}

1. Constitution de la République Démocratique du Congo, telle que révisée par la loi $\mathrm{n}^{\circ} 11 / 002$ du 20 janvier 2011, J.O.RDC, $52^{\text {ème }}$ année, $\mathrm{n}^{\circ} 3 \mathrm{du} 1^{\mathrm{er}}$ janvier 2011.

2. Loi-organique $\mathrm{n}^{\mathrm{o}} 11 / 001$ du 11 janvier 2011 portant composition, attribution et fonctionnement du Conseil Supérieur de l'Audiovisuel et de la Communication.

3. Loi-cadre $n^{\circ} 013 / 2002$ du 16 octobre 2002 sur la télécommunication, J.O.RDC, $\mathrm{n}^{\circ}$ spécial, 25 janvier 2003.

4. Loi $n^{\circ} 96-002$ du 22 juin 1996 fixant les modalités de l'exercice de la liberté de presse, ${ }^{\circ}$ spécial, J.O.RDC, $42^{\text {ème }}$ année, Août 2001

\section{Ouvrages}

1. Charon (J-M.) et Furet (C.), Un secret si bien violé : la loi, le juge et le journaliste, Paris, Seuil, 2000.

2. Charon (J-M.) et Furet (C.), Un secret si bien violé : la loi, le juge et le journaliste, Paris, Seuil, 2000.

3. Eveu (E-N.), Sociologie des mouvements sociaux, La découverte, Paris, 3ème édition.

4. Montesquieu, De l'esprit des lois, Livre III, Chap. V, 94

5. Mugagga (C.) et M'baya (D.), Comprendre les textes juridiques et déontologiques régissant la presse en RDC, Institut Panos Paris, février 2006.

6. Mugagga (C.) et M'baya (D.), Comprendre les textes juridiques et déontologiques régissant la presse en RDC, février 2006, Institut Panos Paris.

7. Mushizi (C-M.), Le cadre juridique et institutionnel des médias congolais, Kinshasa, Décembre 2007.

8. Terrou (F.), Sociologie juridique e droit de l'information, Paris, Librairie Larousse, 1973.

\section{Articles Et revues}

1. Akele Adau (P.), «Dépénaliser les délits de presse en RDC : pourquoi et comment?», in Plaidoyer pour la dépénalisation des délits de presse en République Démocratique du Congo, Kinshasa, mai 2004.

2. Mbuyi (L.), «La problématique de la diffamation et des imputations dommageables par voie de presse », in Code de déontologie des journalistes en RDC, OMC, Kinshasa, février 2005.

3. Usaid et Internews, Revue de la législation sur les Médias en République Démocratique du Congo.

\section{Travail scientifique}

1. Olenga Lumbahee, Dépénalisation des délits de presse en République Démocratique du Congo : analyse de l'action de journaliste en danger (JED). Approche sociologique du droit de l'information, Mémoire de Licence en Journalisme et Politique Intérieure, IFASICKinshasa, 2010.

\section{Autres sources}

1. Journaliste En Danger, La liberté de la presse pendant les élections. Des medias en campagne, Rapport spécial annuel de 2011.

2. Journaliste En Danger, Rapport annuel 2015 : liberté d'expression et élections en RD CONGO, 2016 : L'information sous haute surveillance, Kinshasa, Novembre 2015.

3. Programme de Développement du Secteur Médiatique, Kinshasa, Juin 2012.

4. Code de déontologie et éthique des journalistes congolais.

\section{Webographie}

1. Journaliste En Danger, «L'état de la liberté de la presse en Afrique centrale francophone : la RDC sous prétexte de la guerre », Rapport Annuel, 2012, http://afrikarabia.com/wordpress/wpcontent/uploads/2014/01/JED-Rapport-2012.pdf, consulté le 06 janvier 2016 à 19h30'.

2. Journaliste En Danger, Rapport annuel 2015, in http://www.jed-afrique.org.

3. La liberté de presse, http://www.larousse.fr/encyclopedie/divers/liebrtédelap resse, consulté le 27/01/2016 à 10h30'.

4. Missions de l'UNPC, http://www.unpc.info/article-517.html, consulté le 01 janvier 2016 à 14h30'.

5. République Démocratique Du Congo, Manuel traitant $\mathrm{du}$ droit des médias en Afrique australe, V.2, http//:www.kas.de, consulté le 06 janvier 2016, à 20h30'.

6. Tuti (A.), Dans son rapport annuel 2015 : JED relève 72 atteintes à la liberté de la presse en RDC, http://www.congosynthese.com, consulté 25 avril 2016 à $17 \mathrm{~h} 00$ '.

7. Wikipédia, «La liberté de presse », http://fr.wikipedia.org, consulté le 27/01/2016 à 10h30'.

8. Wikipédia, «Union nationale de la pesse congolais », http://fr.wikipedia.org, consulté le 23 février 2016 à 15h30'. 